\title{
New digital low-level rf system for heavy-ion synchrotrons
}

\author{
Harald Klingbeil, ${ }^{*}$ Ulrich Laier, Klaus-Peter Ningel, Stefan Schäfer, Christof Thielmann, and Bernhard Zipfel \\ GSI Helmholtzzentrum für Schwerionenforschung GmbH, Planckstraße 1, D-64291 Darmstadt, Germany
}

(Received 24 January 2011; published 6 October 2011)

\begin{abstract}
In the scope of the Facility for Antiproton and Ion Research (FAIR) project, several new synchrotrons and storage rings will be built. The existing heavy-ion synchrotron SIS18 has to be upgraded to serve as an injector for the FAIR accelerators. All this imposes new requirements on the low-level rf (LLRF) systems. These requirements include fast ramping modes, arbitrary ion species, and complex beam manipulations such as dual-harmonic operation, bunch merging/splitting, barrier bucket operation, or bunch compression. In order to fulfill these tasks, a completely new and unique system architecture has been developed since 2002, and the system is now used in SIS18 operation. The presentation of this novel system architecture is the purpose of this paper. We first describe the requirements and the design of the LLRF system. Afterwards, some key components and key interfaces of the system are summarized followed by a discussion of technological aspects. Finally, we present some beam experiment results that were obtained using the new LLRF system.
\end{abstract}

DOI: 10.1103/PhysRevSTAB.14.102802

PACS numbers: 29.20.dk, 29.27.-a

\section{REQUIREMENTS AND NECESSITY FOR A NEW LLRF SYSTEM}

The low-level rf (LLRF) system discussed here shall be usable in the existing machines SIS18 (SIS denotes "Schwer-Ionen-Synchrotron," heavy-ion synchrotron) and experimental storage ring (ESR) as well as in the FAIR synchrotrons SIS100 and SIS300 and in the storage rings collector ring (CR), new experimental storage ring (NESR), and accumulator ring (RESR) [1]. These machines perform fast ramp rates of up to $85 \mathrm{MHz} / \mathrm{s}$ and they allow to accelerate a variety of ion species. The large frequency span (e.g. the revolution frequency in SIS18 varies from $215 \mathrm{kHz}$ to $1.36 \mathrm{MHz}$ ) and the arbitrary choice of harmonic numbers are further challenges for the LLRF system.

In order to reach the desired beam quality, a phase accuracy of better than $\pm 3^{\circ}$ and an amplitude accuracy of $\pm 6 \%$ are required. This accuracy has to be reached under dynamic conditions, i.e., during acceleration.

Whereas the LLRF system for SIS18 is located in one rf supply room, this will no longer be possible in SIS100 since the rf cavities are distributed along the circumference $(1084 \mathrm{~m})$. Because of the fast ramp rates and due to the size of the synchrotron, it is therefore impossible to conventionally distribute the rf signals directly via coaxial cables. Cable dispersion, temperature drift, and further deficiencies would lead to a manual calibration effort that would not be manageable.

\footnotetext{
*Permanent address: Technische Universität Darmstadt, 64289 Darmstadt, Germany.

Published by the American Physical Society under the terms of the Creative Commons Attribution 3.0 License. Further distribution of this work must maintain attribution to the author(s) and the published article's title, journal citation, and DOI.
}

Therefore, a new LLRF system has to be developed. It must allow the synchronization of several types of cavities with different quantities (e.g. 14 accelerating cavities, nine bunch compressor cavities, and two barrier bucket cavities in SIS100). Dual-harmonic operation is required in SIS18. Bunch rotation in longitudinal phase space has to be supported by the LLRF system for the SIS18 bunch compressor, the SIS100 bunch compressor, and the CR debuncher. The barrier bucket systems in SIS100 are used to precompress the beam by moving barriers whereas the barrier bucket systems in the NESR shall allow beam accumulation. Also bunch merging and even batch compression scenarios have been discussed for the synchrotrons.

The systems shall allow beam phase control, i.e., damping of coherent longitudinal dipole oscillations that are in phase. Higher-order feedback systems are planned, but they will not be discussed in this document since they may be regarded as stand-alone systems which do not require dedicated rf synchronization with other rf systems.

The flexibility of all these beam manipulation scenarios can only be reached if the new LLRF system is based on digital components. Digital systems allow real-time changes of the operating parameters, and their software may be adapted to new requirements.

\section{DESIGN OF THE LLRF SYSTEM}

In this section, we will describe the overall architecture of the LLRF system. The design includes the abovementioned requirements for FAIR and for the existing synchrotron SIS18.

\section{A. Reference rf signal generation}

Like the synchrotron SIS18, the FAIR synchrotrons will also be fast-ramped machines with large revolution 
frequency spans. Because of the resulting fast frequency changes, it is difficult to distribute rf signals directly from a central location to the different rf systems which are located several hundred meters apart.

Therefore, instead of this direct rf signal distribution, a local generation of variable-frequency reference rf signals was chosen. These reference rf signals are generated by direct digital synthesis (DDS) modules. In this context, one has to make sure that the DDS units are supplied with the same clock signals (constant clock frequency) and that they are fed with the same frequency and/or phase data. The first requirement (same clock signals at different locations) is realized by the bunch phase timing system (BuTiS [2,3]) which distributes delay-compensated fixed-frequency signals from a central place to the supply rooms. The second requirement will be fulfilled by the central control system (CCS [4]) and the White Rabbit timing system [5], which allows one to send control system parameters synchronized with the BuTiS clocks. As a conclusion, we have the possibility to generate synchronized frequency ramps at any location of the facility. This was already successfully tested in the laboratory. In the SIS18 implementation, the BuTiS part of the system has not yet been realized. This was not necessary since all SIS18 rf components are located in the same supply room. Therefore, BuTiS is currently emulated by simply using one local clock source with length-compensated cabling.

The possibility to reproduce synchronized rf signals at different locations has to be extended with respect to the generation of different harmonics in order to allow certain bunch manipulations such as dual-harmonic operation or bunch merging. For these purposes, we mount four DDS units in one crate. Each of these DDS units can be configured with a different harmonic number. Depending on the application, they may typically generate a subset of the following signals in a coherent way: (i) a revolution frequency DDS signal $(h=1$; in this paper, $h$ denotes the harmonic number which determines the ratio $f_{\text {rf }} / f_{0}$ between the $\mathrm{rf}$ frequency and the revolution frequency) which may, e.g., be used to trigger a future barrier bucket system; (ii) an rf signal which is used for normal acceleration ramps (typically at $h=4$ for SIS18); (iii) a second harmonic rf signal $(h=2$ or $h=8)$ for dualharmonic or bunch merging purposes. These rf signals serve as reference signals for the rf cavities. Since each of these reference $\mathrm{rf}$ signals produced by one DDS unit may be used by more than one cavity, we call these DDS modules "group DDS" units (generating "group DDS signals").

Since four group DDS units are located in one crate, they are supplied with the same frequency ramp via a common backplane. This frequency ramp must therefore correspond to the fundamental harmonic $(h=1)$ since the DDS units can then multiply the frequency with the desired $h$ to produce an unambiguous output.

\section{B. Reference rf signal distribution}

Since the group DDS signals can be reproduced at any location, i.e., in any supply room, it is not necessary to transmit them over longer distances. The main purpose of routing group DDS signals is to provide each cavity rf system with the desired reference rf signal. In the most general case, one needs a switch matrix with $n_{i}$ inputs for the group DDS signals (more than four group DDS signals may exist if more than one DDS crate is used). This switch matrix will have $n_{o}$ outputs whose signals may be chosen to be equal to one of the $n_{i}$ inputs. Therefore, each cavity system may be connected to one of the outputs $n_{o}$ (see Fig. 1).

This large switch matrix will be needed for FAIR. It is not yet available, however. For SIS18, we use a configurable distribution amplifier instead. This distribution amplifier has four configurable outputs which can arbitrarily be chosen from four input channels. Therefore, the distribution amplifier is a predecessor of the switch matrix.

The cable lengths in the signal distribution paths have to be equal, of course. This is especially important for the signal path from the cavity to the location where the phase is measured and for the signal path from the group DDS to this phase measurement location.

\section{Cavity synchronization}

Now that one of the group DDS signals can be selected as a reference rf signal for each cavity, it is necessary to lock the phase of the cavity gap signal to that of the selected reference rf signal. This is done by means of a local digital signal processor (DSP) system as shown in Fig. 2.

Each cavity is driven by a local DDS unit which we call "Cavity DDS." The frequency of this cavity DDS must match the frequency of the selected group DDS signal. This is ensured by the CCS which provides both, the group DDS and the cavity DDS units, with compatible frequency ramps. A local DSP system compares the phase of the gap signal with the phase of the reference rf signal [6]. The DSP uses the detected phase difference to modify the frequency of the cavity DDS (closed-loop control algorithm). As a result, the phase of the gap voltage follows the phase of the reference rf signal.

The DSP system is configurable by a variety of registers. This allows one to realize phase offsets or even phase ramps. In the latter case, the phase of the gap signal will always differ from the phase of the reference signal by the desired amount specified by the phase ramp. Constant phase offsets may be used to take the cavity position in the synchrotron into account.

Of course, there are also local cavity control loops such as amplitude control and resonant frequency control. These are not described in this paper, however.

\section{Beam phase control}

The system also allows one to damp coherent longitudinal dipole oscillations of the beam. In [7] it is described 


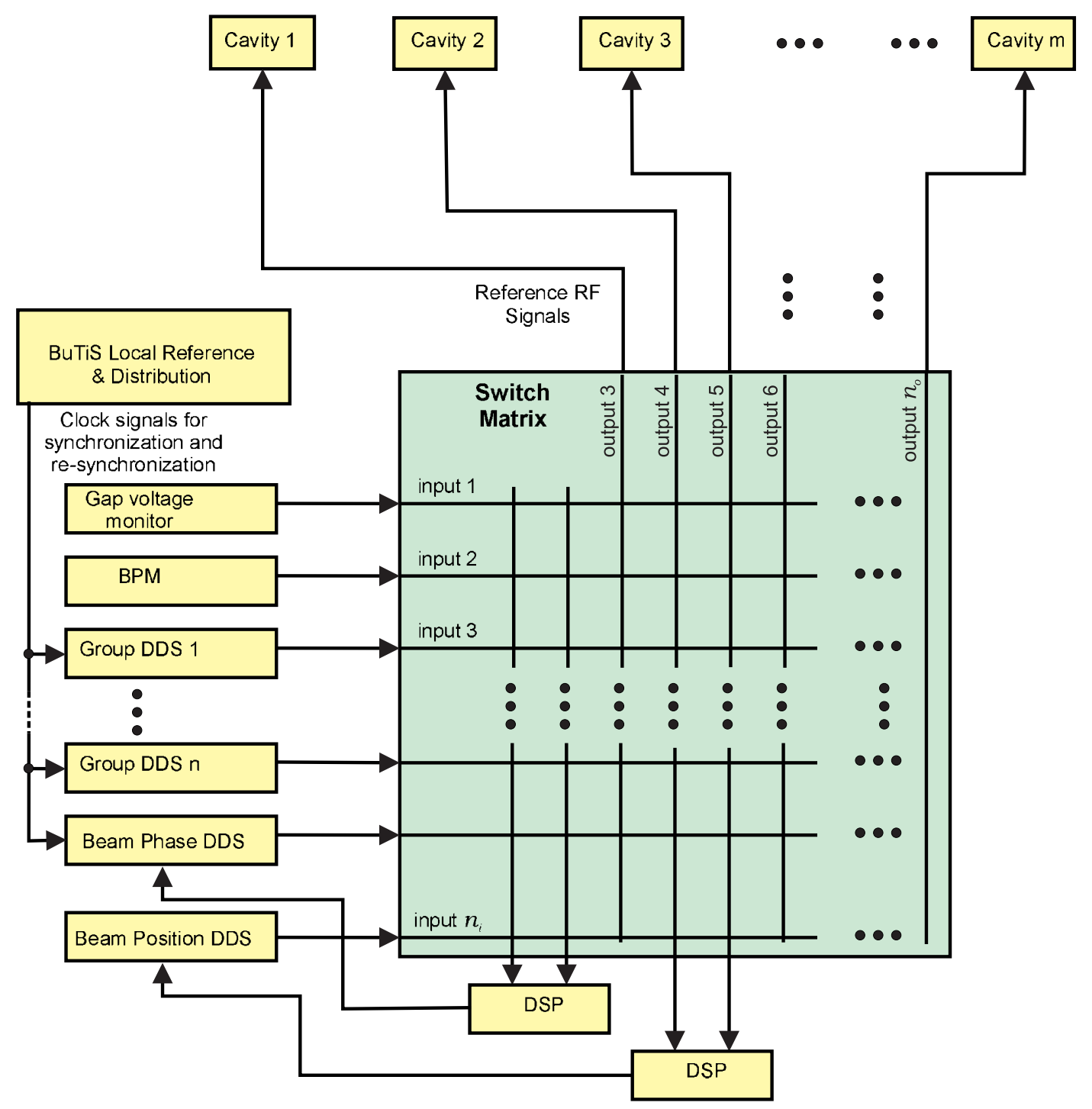

FIG. 1. Routing of group DDS signals to cavity systems.

how this beam phase control is performed with only one cavity. In the scope of the multicavity LLRF system discussed here, beam phase control is realized by a DSP system that compares the phase of the beam signal [delivered by the sum signal of a beam position monitor (BPM), see Fig. 1] with the phase of one gap signal or the vector sum of all gap signals (delivered by the gap voltage monitor, see Fig. 1). The DSP system then tunes the active group DDS in such a way that the bunch oscillation is damped. This group DDS which is used for beam phase control is called "Beam Phase DDS" in Fig. 1. The result is a nested control loop since the local cavity DSP systems receive a reference rf signal that is generated by the higher-level beam phase control. Computer simulations showed that this is feasible if the delays are kept small [8].

One should note that the block diagram in Fig. 1 represents only those LLRF components that are located in one supply room. If more supply rooms are present, they will all have the topology shown in Fig. 1. If beam phase control shall be activated in this case, one has to make sure that all cavities use the same beam phase DDS signal. This will be accomplished by a so-called "inter-DDS communication" which makes sure that passive slave DDS units produce the same output as the active master beam phase DDS. The master DDS sends the calculated frequency word to the slave DDS, and by the means of BuTiS clock slopes, the new frequency will become active at the same time. This leads to an additional delay of up to $10 \mu \mathrm{s}$ which is acceptable according to simulations.

\section{E. Modules and interfaces}

In the scope of the systematic LLRF architecture, the following concepts had a high priority: (i) Modularityeach system component must have a clearly defined functionality. This allows one to reuse the same component in different applications (flexibility). As an example, we 


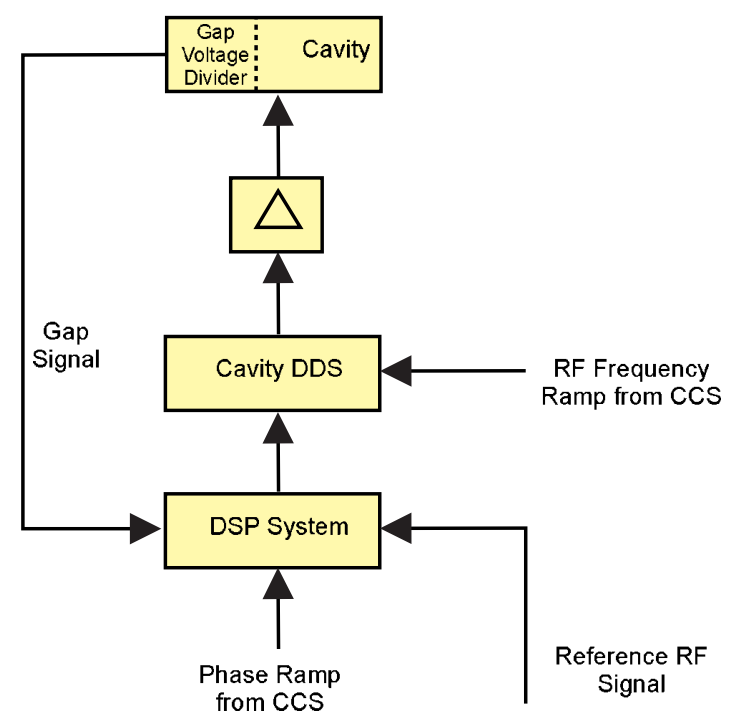

FIG. 2. Phase synchronization of the cavity gap voltage with a reference rf signal.

mentioned before that DDS modules are used as group DDS and as cavity DDS units. Of course, the same type of DDS module is used in both cases. Such a modular DDS unit can, e.g., be configured to run at different harmonic numbers and/or to take external frequency or phase corrections into account. (ii) Clear interfaces-a clearly defined functionality of the modules implies clearly defined interfaces as well. This allows module replacement with units of different functionality or performance (scalability) keeping the rest of the system unchanged.

With respect to the latter point, we mention some important interfaces here: (i) The standard baseband rf signals (50 $\Omega$ system) have a level of $10 \mathrm{dBm}$. They cover a range from $110 \mathrm{kHz}$ (minimum revolution frequency in SIS100) to $5.5 \mathrm{MHz}$ (maximum rf frequency in SIS18). (ii) For slowly varying signals like frequency ramps, amplitude ramps, or phase ramps (bandwidth up to $100 \mathrm{kHz}$ ), different interfaces exist. In the most simple case, they are represented by analog low frequency (LF) signals in the range from $-10 \mathrm{~V}$ to $+10 \mathrm{~V}$. (iii) For routing ramps to digital components (like the DSP system or FPGA-based devices), they may be represented as digital streaming data on a so-called optical direct link (ODL). Modules are available which convert analog ramps into ODL ramps and vice versa. This provides a high flexibility for using the modules. (iv) Components like the DDS unit, the DSP system, or the distribution amplifier have to be configured in real time. For example, the harmonic number of the DDS needs to be changed, the control-loop parameters of the DSP have to be modified, and the routing of the distribution amplifier/switch matrix must be selected. These parameter changes do not consist of a large amount of data, but they have to be processed in real time with minimum delay (microsecond range). On the other hand, several modules need to be configured. Therefore, an optical token ring
(OTR) was defined to fulfill these requirements. Further interfaces exist, but with respect to the LLRF system architecture rf signals, LF signals, ODL, and OTR connections are the most important ones.

\section{REALIZATION IN SIS18}

In SIS18, two ferrite-loaded accelerating cavities (S02BE1 and S08BE2) and a bunch compressor cavity have to be synchronized. Figure 3 shows the resulting block diagram for SIS18. For the sake of simplicity, the bunch compressor system [9] is not shown. It is included in the LLRF topology in the same way as the two ferrite cavities.

In the upper half, one sees the LLRF system for the cavity S08BE2 whereas the lower half shows that for the cavity S02BE1. The blocks denoted as "IFK/FGEN" or "IFK" provide the interface to the central control system where both, ramps and configuration data, are received, respectively.

The crate in the middle denoted as "Frequency Generation" represents the group DDS crate. Two of the four group DDS modules are used to generate local oscillator (LO) signals for the intermediate frequency (IF) processing of the DSP systems as we will describe later.

The crate "S00BE PS Cavity Synchronization" represents the interface to the central control system with respect to all parameter changes. This crate contains a so-called CCS-FIB that will be explained later in the text. The curved lines show the OTR connections to all components whose parameters have to be changed (DDS units, DSP systems, and distribution amplifier).

Further cavities can be added to the block diagram in Fig. 3. For example, three magnetic alloy (MA) loaded accelerating cavities that will work at harmonic number $h=2$ are currently under development [10]. After commissioning, they will be integrated into the LLRF system in the same way.

\section{A. Optical links}

The above-mentioned optical link systems ODL and OTR are based on the same optical fiber system and their telegrams are compatible allowing the same diagnostic tools for both interfaces. Both the OTR and the ODL use the versatile link/hard clad silica optical fiber technology operating at $650 \mathrm{~nm}$.

A proprietary data protocol had to be defined in order to keep the transmission delay as low as possible. For example, in the token ring mode, the telegrams are forwarded by one node to the next one before the whole telegram is received from the previous node. The optical links work with a frequency of $80 \mathrm{MHz}$ and the telegrams consist of 40 bits with Manchester coding (plus 8 bits which determine the token ring device in case of the OTR mode). For streaming data, 32 bit samples are transmitted in one telegram. For parameter changes, 16 bits are used for the address and 16 bits for the data. A header is used to handle 


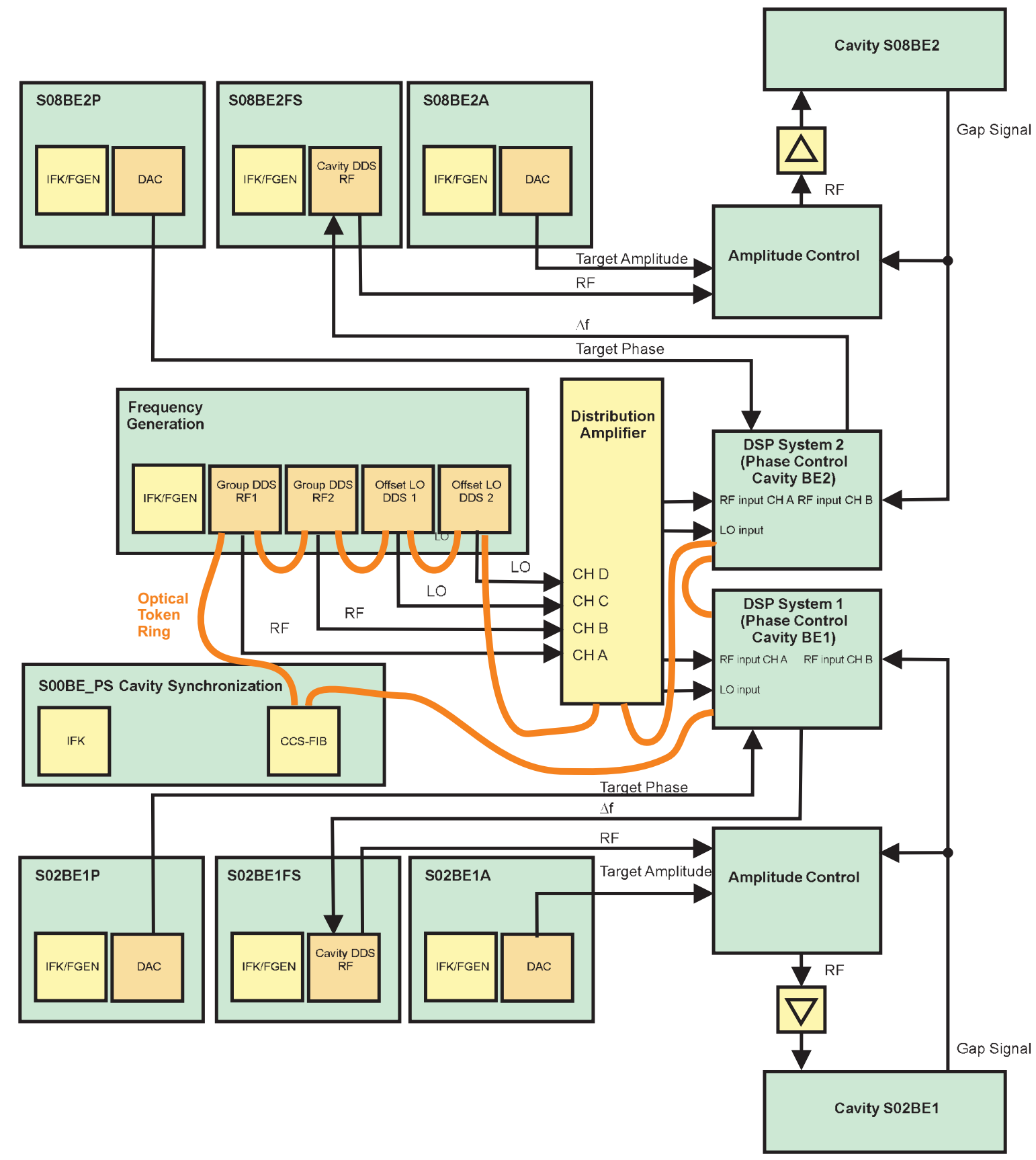

FIG. 3. Block diagram of SIS18 LLRF system.

read and write accesses. In principle, streaming data and register accesses can be used both in OTR and in ODL mode. In the current implementation, however, the ODL is only used for streaming data (ramps), whereas the OTR is only used for parameter changes.

Tests have shown that the optical links may reliably be used for distances up to $20 \mathrm{~m}$ which is sufficient for the supply rooms under consideration. This length refers to the distance from an optical transmitter to an optical receiver. The circumference of an OTR may therefore be much larger.

\section{B. FPGA interface board (FIB)}

The FPGA interface board (FIB) shown in Fig. 4 is a multipurpose PCB. An FPGA selected from Altera's cyclone family is used as a central component. Several interfaces have been standardized (e.g. four optical links are available which may be used as ODLs or as OTRs, and a backplane connector may be used to receive information from the central control system) which allow flexible usage in the following applications: (i) The FIB is used as a motherboard for the DDS unit. In this case, the DDS chip is located on a piggy-back PCB that is plugged onto the 


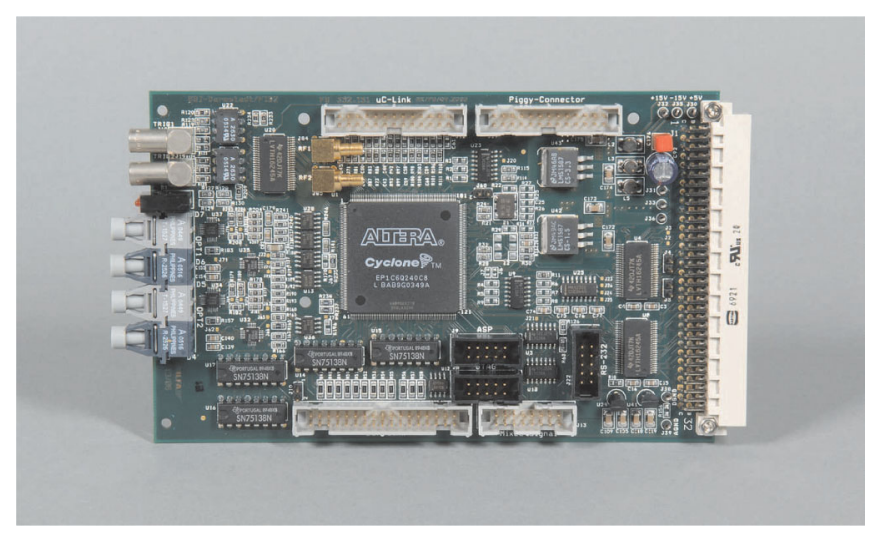

FIG. 4. FPGA interface board (FIB).

FIB. (ii) As the so-called CCS-FIB, it is used to receive parameter changes from the central control system (CCS) and to forward them to the different token ring devices (see Fig. 3). (iii) Many other components have been realized based on the FIB which are not described in this paper.

For the different applications, the same FIB hardware is used, but different firmware codes are necessary. In order to simplify the development, a very high speed hardware description language (VHDL) library was created. This library includes preprogramed VHDL modules (e.g. modules for accessing different types of interfaces or modules for preprocessing of optical link data). All these modules are equipped with a standardized interface called "FPGA universal bus" for interconnection on FPGA level. Complex software can easily be built up by composing different modules.

\section{DSP system}

The term "DSP system" is used to describe an overall system consisting of analog narrowband preprocessing (mixing, filtering), automatic gain control, ADCs, FPGA, DSPs, and DACs. The analog signal preprocessing part works at an IF of $21.4 \mathrm{MHz}$ as described in [6]. In addition to two rf channels, the IF preprocessing system needs an offset-LO signal with the frequency $f_{\mathrm{LO}}=f_{\mathrm{rf}}+f_{\mathrm{IF}}$ which serves as a reference for the two rf signal channels. For the generation of this LO signal, two solutions are currently available: (i) A group DDS is configured in such a way that it not only multiplies the revolution frequency with the desired harmonic number but that it also adds an offset (in this case $f_{\mathrm{IF}}=21.4 \mathrm{MHz}$ ) to the result. In this case, the reference rf signals and the corresponding LO signals are routed to the local cavity DSP systems. (ii) The LO signal is locally derived from the reference rf signal. For this purpose, a digital offset-LO module was developed [11].

The whole digital part including DACs and ADCs is mounted on a peripheral component interconnect (PCI) motherboard in an industrial PC. It uses two Texas Instruments TMS320C6713 DSPs and a XILINX FPGA of the Virtex-II family on an SMT374 TIM module

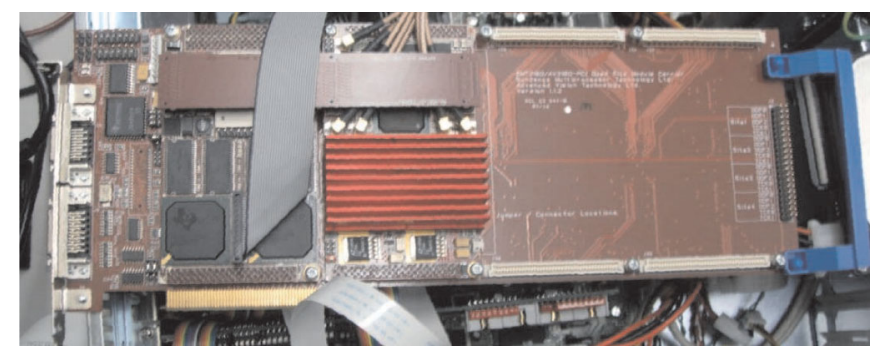

FIG. 5. DSP board.

manufactured by Sundance Multiprocessor Technology Ltd. (see Fig. 5). A customized SMT370 TIM module is used as ADC/DAC board. The whole rf control functionality works in stand-alone mode and only needs the power supply of the PC to operate; the PC is only used for diagnostics purposes.

In contrast to the solution described in [6], the phase and amplitude detection algorithm is no longer implemented as DSP code. It was shifted into the FPGA of the DSP module [12] which allowed us to use DSP processing resources for digital filters and other signal processing.

The DSP system provides a variety of registers for realtime configuration (e.g. control-loop algorithm parameters, filter parameters). In addition, it allows remote data acquisition (e.g. for frequency ramp and phase measurements).

\section{DDS units}

As mentioned before, the DDS unit consists of a FIB motherboard and a DDS piggy-back PCB. The DDS chip AD9854 from Analog Devices Inc. is used. It works at a clock frequency of $200 \mathrm{MHz}$.

The unit (Fig. 6) provides two rf outputs which typically provide in-phase and quadrature signals. External clock inputs are available to allow different modules to be operated using the same clock signals (that will in the future be provided by BuTiS).

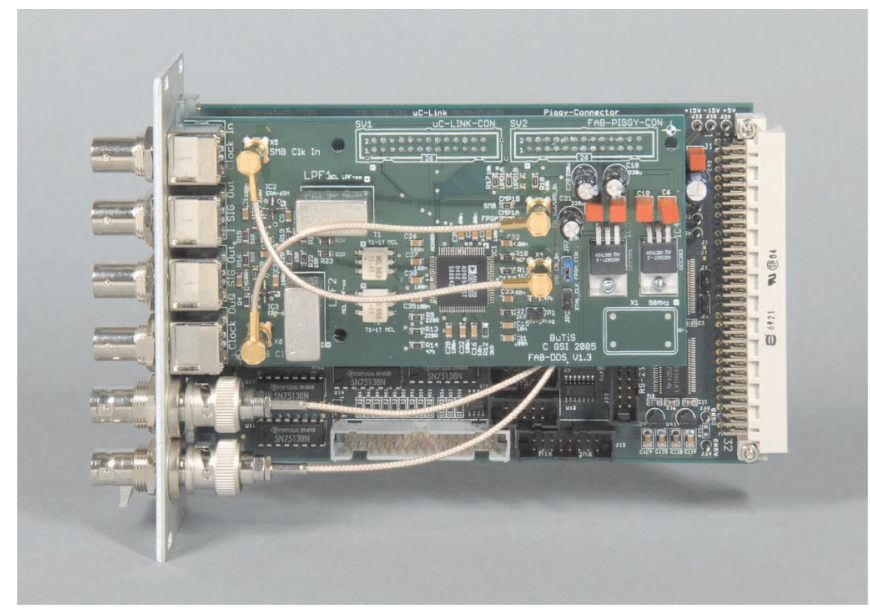

FIG. 6. DDS unit (piggy-back DDS board on FIB). 
Based on the register configuration, the DDS unit provides the following basic features: (i) the frequency ramp is received via the backplane from the CCS; (ii) frequency or phase corrections may be read via ODL; (iii) configuration by register accesses is possible via the OTR or RS232; (iv) the harmonic number may be chosen as a multiplier for the frequency ramp, and a frequency offset may be added; (v) a trigger input is used to initiate certain actions that have to be performed in real time. This can be accomplished by preloading data (e.g. via the OTR) and by sending the trigger pulse afterwards. Further features are available but not of interest in the context of this paper.

\section{E. Operating aspects}

In addition to the pure LLRF functionality, further aspects were taken into account for the practical implementation. A so-called maintenance and diagnostics system was realized [13] which allows operators to perform maintenance and diagnostics actions remotely from an office PC. Firmware files for programable modules are stored on a central server such that a remote firmware update is possible [e.g. via Joint Test Action Group (JTAG)]. Because of logging mechanisms, a recovery of old reliable overall configurations can be done. Furthermore, it is possible to remotely perform data acquisitions, e.g., to see the phase relationships between gap voltages or with respect to the beam signal during standard operation.

\section{MEASUREMENT RESULTS}

In this section, some SIS18 beam experiments are summarized in order to show that the LLRF system works properly and that it allows complex beam manipulations. An optimization of beam dynamics, especially with respect to high-intensity operation, is outside the scope of this paper. Therefore, the experiment results are kept short.

\section{A. Bunch merging}

In order to verify the required flexibility of the LLRF system, a 4:2:1 bunch merging scenario was chosen. The cavity S02BE1 was first operated at $h=4$ with a bunched beam on flattop. Afterwards the cavity S08BE2 was ramped up at $h=2$ while the amplitude of the cavity S02BE1 was reduced. When the S02BE1 amplitude was zero, the harmonic number of that cavity was switched to

TABLE I. Parameters for bunch merging experiment.

\begin{tabular}{lc}
\hline \hline Date of experiment & October 19th, 2010 \\
\hline Ion species & ${ }^{238} \mathrm{U}^{73+}$ \\
Kinetic energy & $300 \mathrm{MeV} / \mathrm{u}$ \\
Particles per cycle & $2 \times 10^{8}$ \\
Revolution frequency $f_{0}$ & $905 \mathrm{kHz}$ \\
Harmonic number $h$ & $4 \rightarrow 2 \rightarrow 1$ \\
Gap voltage & $4 \mathrm{kV} \rightarrow 2 \mathrm{kV} \rightarrow 1 \mathrm{kV}$ \\
\hline \hline
\end{tabular}
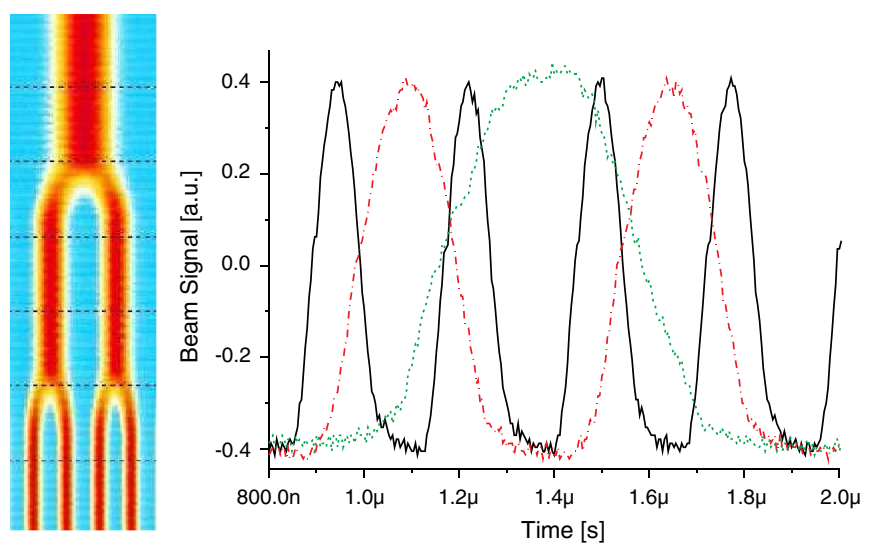

FIG. 7. Waterfall plot (left diagram) and bunch profiles (right diagram) before bunch merging (solid line), after 4:2 bunch merging (dash-dotted line), and after 2:1 bunch merging (dotted line).

$h=1$. As a last step, the S02BE1 amplitude was increased again while the S08BE2 amplitude was decreased to zero.

Slow linear ramps were chosen since the objective of the experiment was not to minimize the bunch merging time or to optimize the beam. Hence, a comparatively low beam intensity in the order of $2 \times 10^{8}$ particles per cycle was used. The relevant parameters of the experiment are summarized in Table I.

Figure 7 shows the bunch profiles that were obtained as BPM sum signals during the $4: 2: 1$ bunch merging process at extraction energy. It is obvious that the bunch merging process worked properly and that symmetrical bunches are obtained.

\section{B. Dual-harmonic operation}

Dual-harmonic experiments were performed at both fixed energy [14] and with acceleration. In the following, we show some results concerning the more challenging acceleration case. The parameters are summarized in Table II.

A comparatively low extraction energy had to be chosen because the limited operating range of the existing ferrite cavities (from $0.8 \mathrm{MHz}$ to $5.5 \mathrm{MHz}$ ) had to be taken into account. In the future, a new MA-based $h=2$ cavity

TABLE II. Parameters for dual-harmonic experiment.

\begin{tabular}{lc}
\hline \hline Date of experiment & March 8 th/9th, 2010 \\
\hline Ion species & ${ }^{238} \mathrm{U}^{73+}$ \\
Ramping speed $\dot{B}$ & $1 \mathrm{~T} / \mathrm{s}$ \\
Injection energy & $11.147 \mathrm{MeV} / \mathrm{u}$ \\
Particles per cycle & $7 \times 10^{8}$ \\
Extraction energy & $110 \mathrm{MeV} / \mathrm{u}$ \\
Revolution frequency $f_{0, \text { inj }}$ & $212 \mathrm{kHz}$ \\
Revolution frequency $f_{0, \text { extr }}$ & $618.75 \mathrm{kHz}$ \\
Harmonic number $h$ & $4 \& 8$ \\
\hline \hline
\end{tabular}



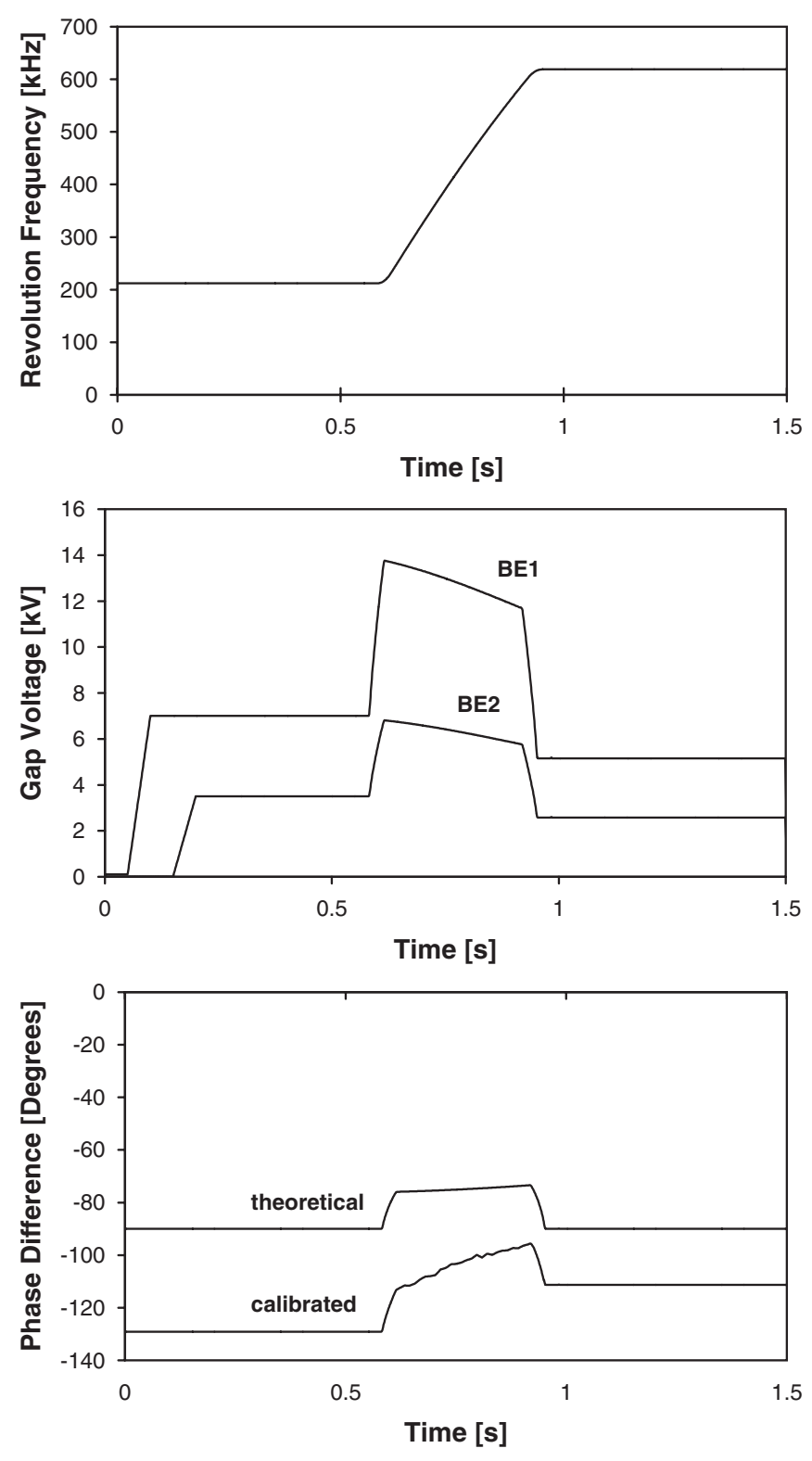

FIG. 8. Ramps for dual-harmonic acceleration (uppermost trace: revolution frequency; trace in the middle: gap voltages; bottommost trace: phase difference).

system (consisting of three independent units) will be installed in SIS18 [10] such that dual-harmonic operation will be performed with $h=2 / 4$.

Figure 8 shows the ramps which were used to realize the dual-harmonic use case. The cavity S08BE2 was operated at $h=4$, the cavity S02BE1 at $h=8$. The amplitude at $h=4$ under stationary conditions was twice as high as the second harmonic one at $h=8$. The phases were defined in such a way that in the stationary case a phase difference of $-90^{\circ}$ corresponds to a negative zero crossing of the second harmonic voltage with respect to the positive zero crossing of the first harmonic (longitudinal defocusing in bunch center, saddle point). In the last diagram in Fig. 8, two

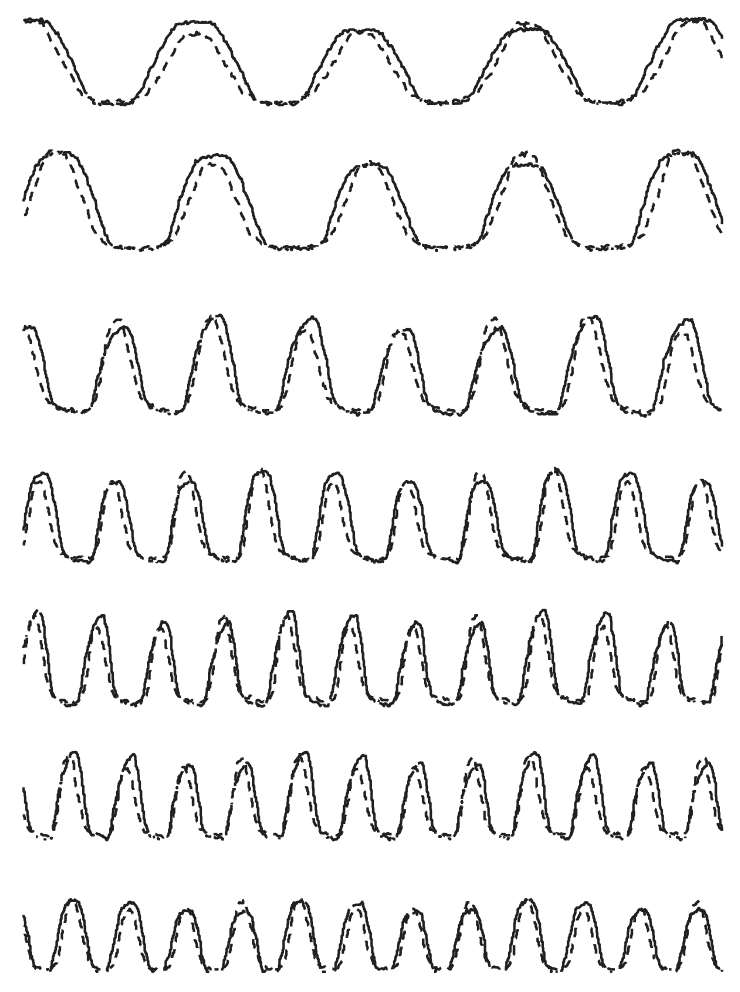

FIG. 9. Bunch profiles during acceleration process for singleharmonic acceleration (dashed line), and dual-harmonic acceleration (solid line). In the horizontal direction, the time scale is in the order of microseconds whereas several traces are omitted in the vertical direction such that several tens of milliseconds are visible.

curves representing the phase difference are shown. The upper one is the theoretical curve, and the lower one is obtained if a phase calibration is performed. The phase calibration is required especially to compensate for cable length differences and other delays. This phase calibration only depends on the frequency. Also the gap voltage amplitudes had to be calibrated. This calibration depends on both frequency and amplitude. It is not shown in the diagram, however.

Figure 9 shows the development of the bunch profile during the experiment. One clearly sees the desired bunch flattening effect because the bunch length of the dualharmonic case is larger than that of the single-harmonic case. Please note that the two measurements correspond to different machine cycles such that slightly different beam intensities were present. Therefore, the bunch height of the single-harmonic case is not comparable with that of the dual-harmonic case. During portions of the ramp, the bunches are symmetrical, at other times a slope is visible. This is due to an imperfect manual calibration and will be improved in the future by automatic calibration processes. Such a calibration process includes a correction of the gap voltage depending on both, the gap voltage target value and the frequency. The phase only needs to be corrected depending on the frequency. 


\section{Accuracy}

Fixed energy dual-harmonic experiments [14] as well as the bunch merging experiment showed that a phase accuracy of better than $\pm 3^{\circ}$ has to be achieved if bunches without visible asymmetry shall be generated. They also showed that the amplitude accuracy must be better than $6 \%$ to reach this goal. These observations are in compliance with the design parameters of the FAIR LLRF system.

\section{SUMMARY AND OUTLOOK}

LLRF architecture considerations to fulfill the requirements for FAIR (especially multiharmonic operation) were presented. A detailed LLRF topology was introduced. Flexibility, modularity, scalability, clearly defined interfaces, and maintenance and diagnostics issues are important design aspects. The technological implementation for the existing SIS18 synchrotron was described based on stateof-the-art digital signal processing. Optical links with a suitable protocol were presented as interfaces for lowdelay high-reliability data transmission. Key components like the DDS unit for flexible rf and LO signal generation, the DSP system for narrowband high-accuracy real-time phase and amplitude detection as well as for the controlloop algorithm implementation, and the FPGA boards as multipurpose interfacing devices were presented.

Two use cases were discussed in detail in order to verify the topology as well as the technical implementation. The measurement results show that the system works properly with the required accuracy.

The development of the LLRF system has to continue even though the architecture will not change in principle. The functionality of several components still has to be extended and for some components the performance has to be improved. The timing system (White Rabbit, BuTiS) integration and the FAIR control system integration are still ongoing. The gap voltage amplitudes and phases have to be corrected by an automatic calibration procedure in the future (this was done manually during the beam experiments). Finally, the beam manipulation processes have to be optimized for high beam currents. This, however, will only be feasible with acceptable effort after the automatic calibrations mentioned before and the integration into the central control system are completed since different operating conditions can then be tested more easily.

\section{ACKNOWLEDGMENTS}

The authors would like to thank M. Bousonville, M. Kumm, D. Mondry, P. Moritz, M. Sam, S. Sanjari, H. Veldman, T. Winnefeld, and T. Wollmann for many technical contributions to the overall LLRF system. We are also grateful for the help of several persons from different GSI departments (e.g. Accelerator Electronics, Experiment
Electronics, Operating, and Synchrotrons). Furthermore, the authors thank P. Hülsmann, H.G. König, and G. Schreiber for many fruitful discussions. In addition we would like to thank our external collaboration partners who were and still are dealing with specific problems in detail (e.g. Professor M. Glesner, Technical University Darmstadt; Professor K. Fricke-Neuderth, University of Applied Sciences Fulda; Professor P. Zipf, University Kassel; and the industrial partners Sundance Ltd., 3L Ltd., LogiTrue, UbiSys). We thank the reviewers for their helpful comments and we are grateful to R. Pasquinelli for proofreading the manuscript.

[1] FAIR Baseline Technical Report, 2006, Accelerator and Scientific Infrastructure.

[2] P. Moritz, GSI Annual Report 2006, FAIRACCELERATORS-20, 2006.

[3] M. Bousonville and J. Rausch, in Proceedings of HIAT, Venezia, Italy, 2009 [http://accelconf.web.cern.ch/ accelconf/HIAT2009/doc/1_HIAT_committees.pdf].

[4] R. C. Baer, Technical Report March 2009, presentation for FAIR Machine Advisory Committee, https://indico.gsi.de.

[5] J. Serrano, P. Alvarez, M. Cattin, E. G. Cota, J. Lewis, P. Moreira, T. Wlostowski, G. Gaderer, P. Loschmidt, J. Dedič, R. Baer, T. Fleck, M. Kreider, C. Prados, and S. Rauch, in Proceedings of the 12th International Conference on Accelerator and Large Experimental Physics Control Systems, Kobe, Japan, 2009 [http://accelconf.web.cern.ch/AccelConf/icalepcs2009/ papers/tuc004.pdf].

[6] H. Klingbeil, IEEE Trans. Instrum. Meas. 54, 1209 (2005).

[7] H. Klingbeil, B. Zipfel, M. Kumm, and P. Moritz, IEEE Trans. Nucl. Sci. 54, 2604 (2007).

[8] C. Spies, P. Zipf, M. Glesner, and H. Klingbeil, IEEE Workshop on Rapid System Prototyping, 2008, p. 196.

[9] P. Huelsmann, G. Hutter, and W. Vinzenz, in Proceedings of the 9th European Particle Accelerator Conference, Lucerne, 2004 (EPS-AG, Lucerne, 2004), pp. 1165-1167.

[10] P. Huelsmann, R. Balss, H. Klingbeil, U. Laier, K.-P. Ningel, C. Thielmann, and B. Zipfel, in Proceedings of the IPAC'10 Conference, Kyoto, Japan (ICR, Kyoto, 2010), pp. 744-746.

[11] M. Kumm, H. Klingbeil, and P. Zipf, IEEE Trans. Circuits Systems, Part I 57, 2487 (2010).

[12] A. Guntoro, P. Zipf, O. Soffke, H. Klingbeil, M. Kumm, and M. Glesner, in International Workshop on Applied Reconfigurable Computing (ARC2006), Delft, The Netherlands, 2006 [http://www.arc-symposium.org/ $\operatorname{arc2006/].~}$

[13] H. Klingbeil, K.-P. Ningel, and M. Kumm, GSI Annual Report 2007, GSI-ACCELERATORS-13, 2007.

[14] K.-P. Ningel, P. Huelsmann, H. Klingbeil, U. Laier, C. Thielmann, and B. Zipfel, in Proceedings of the IPAC'10 Conference, Kyoto, Japan (Ref. [10]), pp. 1410-1412. 